\title{
Enhancing Patient-Centered Care: SGIM and Choosing Wisely
}

\author{
Laurence F. McMahon Jr, MD, MPH', 2,17, Rebecca Jennifer Beyth, MD', Alfred Burger, MD \\ Vineet Chopra, MD, MSC ${ }^{1,5,17}$, David Feldstein, $M D^{6}$, Deborah Korenstein, $M D^{7}$, \\ Usha Subramanian, $M D^{8}$, Jeremy Sussman, $M D, M S c^{1,5,17}$, Brent Petty, $M D^{9}$, and Jeff Tice, $M D^{10}$
}

'Division of General Medicine, Ann Arbor, MI, USA; ${ }^{2}$ Department of Health Management and Policy, University of Michigan, Ann Arbor, MI, USA; ${ }^{3}$ Department of Internal Medicine, University of Florida, Malcom Randall VA Medical Center, Gainesville, FL, USA; ${ }^{4}$ Department of Internal Medicine, Beth Israel Medical Center, Albert Einstein College of Medicine, New York City, NY, USA; ${ }^{5}$ Ann Arbor VA Medical Center, Ann Arbor, MI, USA; ${ }^{6}$ Department of Internal Medicine, University of Wisconsin, Madison, WI, USA; ${ }^{7}$ American College of Physicians, Philadelphia, PA, USA; ${ }^{8}$ Department of Internal Medicine, Indiana University, Indianapolis, IN, USA; 'Department of Internal Medicine, Johns Hopkins University, California, MD, USA; ${ }^{10}$ Department of Internal Medicine, University of California, San Francisco, San Francisco, CA, USA;

"Department of Internal Medicine, University of Michigan, Ann Arbor, MI, USA.

J Gen Intern Med 29(3):432-3

DOI: $10.1007 / \mathrm{s} 11606-013-2617-8$

(c) Society of General Internal Medicine 2013

$\mathrm{O}$ ne of the touchstones of academic General Internal Medicine, whether practiced in an ambulatory care setting or at the patient's bedside, is its focus on enhancing and teaching patient-centered care. In an era of increasing cost consciousness, patient centeredness requires thoughtful, ongoing discussions about the risks and benefits of a myriad of medical tests and procedures. Against this backdrop, the Society of General Internal Medicine (SGIM) chose to participate in the American Board of Internal Medicine Foundation's Choosing Wisely initiative.

The Choosing Wisely initiative aims to promote conversations between patients and their physicians around tests and procedures whose necessity should be questioned and whose potential harms and benefits clarified within the context of each patient's care plan. To date, 54 medical societies and 17 consumer groups have identified 280 potentially unnecessary medical tests and treatments that are commonly performed in the United States. ${ }^{1}$ To further assist patients in engaging with their physicians in discussions about these tests and procedures, Consumer Reports is partnering in this process to develop and disseminate materials for patients through large, publically oriented consumer groups. This alliance of science, advocacy and policy is ultimately designed to improve patient care and clinical outcomes, and reduce wasteful and unnecessary healthcare expenditures.

In discussing health care costs and the Choosing Wisely approach, Castle and Guest from the American Board of Internal Medicine (ABIM) Foundation note, "The initial focus should be on overuse of medical resources, which not only is a leading factor in the high level of spending on healthcare but also places patients at risk of harm." estimates suggest that as much as $30 \%$ of all healthcare

Published online October 3, 2013 spending is wasteful. ${ }^{3}$ Volpp and colleagues also identify the promise of the Choosing Wisely initiative when they note, "The Choosing Wisely initiative represents an important first step toward the identification of low-value services, more meaningful because it is a step taken jointly by consumer groups and professional societies." They go on to note, "Given fiscal realities, reducing low-value services is what will allow continued support for the coverage of high-value services." 4

The ad hoc SGIM Choosing Wisely Committee was selected from the existing SGIM Clinical Practice Committee, and the Evidence-based Medicine Task Force, as well as selected society members. The goal of the ad hoc committee was to identify important topics for academic general internal medicine faculty across the spectrum of clinical practice. The committee operated under a few notable constraints. First, there had to be existing evidence on the selected topic, as there was no opportunity to construct new systematic reviews or evidence syntheses as part of the process. Second, the Choosing Wisely initiative was limited to five topics or recommendations.

Given these operating parameters and the interest in selecting topics across the spectrum of academic practice, the committee began by internally soliciting topics for consideration. These topics were then circulated to all committee members and an initial vote was taken to identify the top ten topics. Subsequently, the committee sought to establish the evidence base for each of the ten topics, and then conducted a conference call to determine relevant domains through which to weigh the selection of the final five topics. The agreed-upon relevant domains included: the strength of the evidence-base for that particular recommendation; our "standing" on the topic as general internists; the number of patients likely to be affected by the recommendation; the financial impact of the topic; the relative cost effectiveness of the intervention; and potential harm to the patient. The top ten topics were then scored across these multiple domains to determine five final recommendations for development as SGIM Choosing Wisely topics.

Each of the final five topics was internally assigned to two committee members who developed the one sentence 
summary statement and the three to four line summary paragraph that are the published products, with accompanying references, for the American Board of Internal Medicine Foundation's Choosing Wisely website. In addition, the committee produced a more thorough one-page summary of each topic, with an expanded reference list to more completely describe the studies and rationale behind the recommendations. These summary statements were then circulated to all of the ad hoc Choosing Wisely SGIM committee members for internal peer review and adjudication. These more complete descriptions are published on the JGIM website (www.jgim.org) for review and dissemination to the SGIM membership and beyond.

It is clear that a different committee might arrive at other topics that would be of equal merit for inclusion in this initiative. The list that was chosen is therefore not meant to represent the optimal choices, but rather the selection by this committee, given the charge and relevant constraints. We encourage all JGIM readers to become familiar with the
Choosing Wisely website, and to continue to actively engage their patients in meaningful conversations regarding the risks and benefits of tests and procedures and to engage learners in the importance of these discussions.

Corresponding Author: Laurence F. McMahon, Jr, MD, MPH; Division of General Medicine, 2800 Plymouth Road Building 16, Room 430W. Ann Arbor, MI 48109, USA (e-mail: Imcmahon@med.umich.edu).

\section{REFERENCES}

1. Choosing Wisely. An Initiative of the ABIM Foundation. http://www. choosingwisely.org, accessed 8/15/13

2. Cassel CK, Guest JA. Choosing wisely: helping physicians and patients make smart decisions about their care. JAMA. 2012;307(17):1801-2.

3. Berwick DM, Hackbarth A. Eliminating waste in US health care. JAMA. 2012;307(14):1513-6.

4. Volpp KG, Loewenstein G, Asch DA. Choosing wisely Low-value services, utilization and patient cost sharing. JAMA. 2012;308(16):1635-6. 